\title{
Estado vacunal y motivos de no vacunación contra el virus del papiloma humano en adolescentes admitidas en el Hospital Pediátrico del Centro Hospitalario Pereira Rossell
}

\author{
Martín Notejane*, Carlos Zunino ${ }^{\dagger}$, Dahiana Aguirre ${ }^{\ddagger}$, Paula Méndez ${ }^{\ddagger}$, \\ Loreley García§, Walter Pérez
}

\section{Resumen}

Introducción: en Uruguay se ofrece gratuitamente una vacuna tetravalente contra el virus del papiloma humano (VPH) a todas las adolescentes de 12 años o mayores. Las coberturas logradas no han sido las esperadas.

Objetivo: describir el estado vacunal contra el VPH, motivos de no vacunación y reporte de efectos adversos en las adolescentes hospitalizadas en un centro de referencia en Uruguay.

Material y método: estudio descriptivo mediante encuesta anónima a adolescentes de 12 años o mayores hospitalizadas en cuidados moderados del Hospital Pediátrico del Centro Hospitalario Pereira Rossell durante el segundo semestre de 2016. Se excluyeron las adolescentes que se negaron a participar, las portadoras de retardo mental o pasibles de cuidados paliativos. Se analizó: edad, estado vacunal contra VPH, motivos de no vacunación y de rechazo a la vacuna, efectos adversos. Fue aprobado por el Comité de Ética de la institución.

Resultados: se encuestaron 112 adolescentes. Edad media: 13,5 años (12-14,6). Reportaron recibir al menos una dosis de la vacuna contra VPH: 45/112 (40,1\%). Motivo de no vacunación más frecuente reportado: desconocimiento de la existencia de la vacuna: 48/67 (71,6\%), seguido de rechazo o negativa de la adolescente 0 adulto responsable: 13/67 (19,4\%). El principal motivo de rechazo a recibir la vacuna fue la falta de información: 7/13. No se registraron efectos adversos graves.

Conclusiones: se observó una cobertura vacunal similar a datos nacionales. El conocimiento y la información de las adolescentes y sus cuidadores sobre esta vacuna fueron los principales motivos de no vacunación detectados.

Palabras clave: VACUNAS CONTRA PAPILOMA VIRUS ADOLESCENTE

Key words: $\quad$ ADOLESCENTS

VACCINES

HUMAN PAPILLOMA VIRUS

\footnotetext{
* Asistente de Clínica Pediátrica. Departamento de Pediatría. Facultad de Medicina. Universidad de la República. Montevideo, Uruguay. † Profesor Adjunto de Pediatría. Departamento de Pediatría. Facultad de Medicina. Universidad de la República. Montevideo, Uruguay. ¥ Residente de Pediatría. Departamento de Pediatría. Facultad de Medicina. Universidad de la República. Montevideo, Uruguay.

$\S$ Profesora Agregada de Pediatría. Departamento de Pediatría. Facultad de Medicina. Universidad de la República. Montevideo, Uruguay. I Profesor de Clínica Pediátrica. Departamento de Pediatría. Facultad de Medicina. Universidad de la República. Montevideo, Uruguay. Declaramos no tener conflicto de interés.

Trabajo inédito.

Correo electrónico: mnotejane@gmail.com

Recibido: 6/11/17.

Aprobado: 16/2/18
} 


\section{Introducción}

En Uruguay, el cáncer de cuello de útero (CCU) constituye la tercera neoplasia en frecuencia en mujeres. Entre los años 2009 y 2013 la tasa de mortalidad fue de cinco cada 100.000 mujeres entre los 30 y 34 años, llegando a 14,2 cada 100.000 entre los 50 y 54 años $^{(1)}$. Esta enfermedad se encuentra asociada a la infección por variantes oncogénicas del virus del papiloma humano (VPH), siendo los serotipos 16, 18 y 45 responsables de la mayoría de los casos en Uruguay ${ }^{(2)}$. La infección causada por VPH se ha incrementado de forma alarmante en los últimos años y las tasas de mayor prevalencia se observan en adolescentes y mujeres jóvenes ${ }^{(3,4)}$. La infección por serotipos oncogénicos de VPH es causante también de una proporción importante de cánceres de orofaringe, vagina, vulva y pene ${ }^{(5,6)}$.

La prevención primaria de la infección por el VPH consiste en educación e inmunización universal contra las variantes oncogénicas de este virus. El uso de preservativos o condón previene hasta $70 \%$ de las infecciones. La prevención secundaria se realiza por medio de la detección temprana de lesiones que pudieran favorecer la carcinogénesis ${ }^{(7,8)}$. El método de tamizaje o screening utilizado en forma generalizada es el estudio citológico cervicovaginal (PAP), útil para la detección de lesiones premalignas con el objetivo de un tratamiento precoz. Este cribado debe hacerse aun en personas vacunadas ${ }^{(9)}$. En nuestro país se solicita el PAP a partir de los 21 años de edad siguiendo las recomendaciones internacionales ${ }^{(10)}$.

En Uruguay, desde el año 2013 el Ministerio de Salud (MS), en el marco del Plan Nacional de Prevención Integral del CCU, comenzó a ofrecer de forma gratuita una vacuna contra VPH tetravalente a todas las adolescentes nacidas a partir del año 2001, realizando en el año 2014 una campaña de nivelación para la cohorte 2000. El esquema de administración fue cambiado de tres a dos dosis en las menores de 15 años en el año 2017. Desde el inicio de la vacunación al 31 de diciembre de 2016 la cobertura promedio fue de $46 \%$ de la población objetivo*. Si bien desde el MS se han ejecutado estrategias para el aumento de las coberturas (vacunación en centros educativos, entre otras), el porcentaje de vacunación no ha sido el esperado. Existen numerosas explicaciones a la vacilación frente a la vacuna contra el VPH: escasa información de las adolescentes y sus familias, creencias religiosas, falsos rumores de efectos adversos graves, existencia de grupos cuestionadores de vacunas, etcétera ${ }^{(11)}$. Sin embargo, no hay estudios nacionales pu-

* Datos proporcionados por la División de Epidemiología del Ministerio de Salud, Uruguay. blicados que aborden esta temática. La información y el conocimiento que pueden aportar este tipo de investigaciones son importantes para el diseño y las estrategias de implementación de políticas públicas para la prevención del CCU mediante vacunación.

\section{Objetivo}

Describir el estado vacunal contra el VPH y los motivos de no vacunación de adolescentes hospitalizadas en un centro de referencia de Uruguay durante el segundo semestre del año 2016.

\section{Material y método}

Se realizó una encuesta anónima con preguntas cerradas a adolescentes de 12 años o mayores, hospitalizadas en salas de cuidados moderados del Hospital Pediátrico, Centro Hospitalario Pereira Rossell. Se calculó una muestra considerando las hospitalizaciones en el segundo semestre del año anterior (161 adolescentes)*, asumiendo una cobertura vacunal de $40 \%$ y un intervalo de confianza de $95 \%$. El número de adolescentes necesario a encuestar fue 112. Las encuestas fueron realizadas a partir del primero de julio de 2016 de forma diaria hasta alcanzar el número a encuestar calculado.

Se excluyeron las adolescentes que se negaron a participar y aquellas con diagnóstico de discapacidad intelectual o una condición de salud pasible de cuidados paliativos.

Se registró: edad (años) y motivo de hospitalización. Se indagó estado vacunal contra el VPH (sí o no) y número de dosis recibidas. La información sobre el estado de vacunación se recabó a través del relato de la adolescente $\mathrm{o}$ adulto referente, sin corroborar los datos con el carné de vacunas, ni con el registro nominal nacional.

Se analizaron los motivos de no vacunación: desconocimiento de la existencia de la vacuna, no recomendación por parte de los profesionales de la salud, rechazo a esta vacuna por parte de la adolescente o los adultos referentes, no disponibilidad de la vacuna, presencia de contraindicaciones, u otros motivos. De las que refirieron rechazar la vacuna, se indagaron las razones: falta de información sobre la vacuna, dudas sobre su seguridad, dudas sobre su efectividad, otros. En caso de recibir la vacuna, se indagó sobre eventos adversos supuestamente atribuidos a la vacunación (ESAVIs). Se catalogaron como graves si requirieron hospitalización o amenazaron la vida.

Se realizó un cuestionario confeccionado por los autores con la finalidad de recopilar los datos. Se aplicó

* Dato proporcionado por el Departamento de Registros Médicos de la institución. 
Tabla 1. Motivos de hospitalización de las adolescentes encuestadas $(n=112)$.

\begin{tabular}{lcc}
\hline Motivo de la hospitalización & $F A$ & $F R(\%)$ \\
\hline Patología psiquiátrica & 46 & 41 \\
Patología quirúrgica & 31 & 27,7 \\
Motivos sociales & 15 & 13,4 \\
Patología aguda no infecciosa & 9 & 8 \\
Patología aguda infecciosa & 7 & 6,25 \\
Procedimientos o estudios & 4 & 3,6 \\
\hline
\end{tabular}

FA: frecuencia absoluta; FR: frecuencia relativa.

una prueba piloto para evaluar la comprensión del cuestionario por parte de las adolescentes.

\section{Análisis estadístico}

Las variables cualitativas se expresaron en frecuencias absolutas y relativas (\%), las variables cuantitativas en medidas de tendencia central y su rango. Para procesar los datos se utilizó el programa Epi Info ${ }^{\mathrm{TM}}$ versión 7.2.

\section{Aspectos éticos}

Este estudio contó con la aprobación de la dirección y del Comité de Ética en Investigación de la institución. Se solicitó asentimiento a las adolescentes y consentimiento informado a sus cuidadores, respetando en todo momento la confidencialidad de los datos. Se ofreció la posibilidad de contestar la encuesta a solas o acompañada de un familiar.

\section{Resultados}

La encuesta fue contestada por 112 adolescentes que cumplían los criterios de inclusión. La media de edad fue de 13,5 años (12-14,6 años). El principal motivo o causa de hospitalización fueron las patologías psiquiátricas, tales como intentos de autoeliminación, trastornos del humor o del comportamiento y consumo problemático de sustancias psicoactivas. En la tabla 1 se presentan los motivos de hospitalización de la población de adolescentes incluidas en el estudio.

De las adolescentes encuestadas, 45/112 (40,1\%) reportaron haber recibido al menos una dosis de la vacuna contra el VPH; 31/45 presentaban el esquema completo.

El motivo de no vacunación más frecuente reportado fue el desconocimiento de la existencia de esta vacuna: $48 / 67$ (71,6\%). En la tabla 2 se expresan los motivos por los cuales no se vacunaron contra el VPH.

El principal motivo reportado de rechazo o negativa a recibir la vacuna fue la falta de información: 7/13; se-
Tabla 2. Motivos de no vacunación contra el virus del papiloma humano referidos por las adolescentes encuestadas $(n=67)$.

\begin{tabular}{lcc}
\hline Motivos referidos & FA & FR (\%) \\
\hline $\begin{array}{l}\text { Desconocimiento de la existencia de la vacuna } \\
\text { Rechazo o negativa de la adolescente }\end{array}$ & 48 & 71,6 \\
y/o adulto referente & 13 & 19,4 \\
Olvidos & 2 & 3 \\
Falsas contraindicaciones para la vacuna & 2 & 3 \\
Contraindicación & 1 & 1,5 \\
Falta de disponibilidad de la vacuna & 1 & 1,5 \\
\hline FA: frecuencia absoluta; FR: frecuencia relativa. & & \\
\hline
\end{tabular}

guido de dudas sobre su seguridad: $4 / 13$, y dudas sobre la efectividad: $2 / 13$.

En relación con los ESAVIs: 44/45 no reportaron ninguno. Una adolescente reportó dolor y edema local posvacunación, catalogado como no grave.

\section{Discusión}

En este estudio la cobertura de vacunación contra VPH fue de $40 \%$, si bien no se trata de una población representativa del total de adolescentes de Uruguay, coincide con los datos de cobertura a nivel nacional, sustancialmente menor al compararla con la cobertura promedio de las vacunas incluidas en el certificado de esquema de vacunación (aproximadamente 95\%)*. Por esta razón, cobra importancia conocer los motivos de no vacunación contra el VPH en esta población.

Los datos de cobertura de vacunación contra VPH en la región son heterogéneos (40\%-80\%). Estas diferencias pueden tener múltiples explicaciones: diferentes políticas de inmunización de cada país (obligatoriedad, vacunación en centros educativos, esquema utilizado y estrategias de comunicación), programas de educación sexual y reproductiva, influencias culturales, entre otros ${ }^{(12,13)}$.

Un elemento importante en las coberturas vacunales es la aceptabilidad que determinada vacuna tenga en la población. La población objetivo de la vacuna contra el VPH (adolescentes y mujeres jóvenes) plantea ciertas consideraciones a tener en cuenta entre ellas: aspectos psicosociales de esta etapa vital, rol de los adultos refe-

\footnotetext{
* Datos proporcionados por la División de Epidemiología del Ministerio de Salud, Uruguay.
} 
rentes y la consideración de la autonomía en aquellos adolescentes competentes para la toma de decisiones ${ }^{(14)}$.

Son múltiples los factores involucrados en la aceptabilidad de una vacuna: el conocimiento general de su utilidad, percepción del riesgo y gravedad de enfermar, percepción de la seguridad de la vacuna, edad de la vacunación, fuente de la recomendación, costo, otros ${ }^{(14)}$. Existe vasta literatura internacional que analiza estos factores involucrados, no así a nivel nacional.

Esta comunicación presenta como principal limitante el sesgo de selección de la población encuestada. La institución prestadora de salud donde se realizó la investigación asiste a usuarios del subsector público procedentes principalmente de la capital del país y área metropolitana, de zonas con gran vulnerabilidad social y cultural. Sin embargo, este estudio permitió identificar la existencia de barreras que deberían incluirse en la planificación de políticas de vacunación dirigidas a estas usuarias. Sería oportuno poder replicar este trabajo en otros prestadores de salud incluyendo una muestra representativa de todo el país o diseñar estudios cualitativos que indaguen creencias de las adolescentes y sus familiares sobre la vacunación contra el VPH. Asimismo, sería oportuno considerar estos aspectos según el nivel de escolaridad de las adolescentes y nivel de instrucción de los adultos referentes.

En esta serie el motivo de no vacunación más frecuente reportado fue el desconocimiento de la existencia de esta vacuna. Esto podría estar reflejando la falta de accesibilidad real a la vacuna a pesar de su disponibilidad en todos los vacunatorios del país en forma gratuita. Para que se pueda garantizar y ejercer el derecho a la vacunación el primer paso es dar a conocer la existencia de este recurso preventivo. Asimismo, debe ir acompañado de políticas de información y sensibilización que involucren a todo el equipo de salud. La hospitalización de adolescentes debe ser uno de los escenarios propicios para esta tarea.

Entre las adolescentes que conocían la existencia de la vacuna, la falta de información fue el motivo más frecuente de rechazo a la misma. La información es requisito necesario y obligatorio para que las personas puedan decidir en forma responsable sobre sus problemas de salud. El equipo de salud juega un rol importante a la hora de brindar información en relación con las vacunas en todos los niveles de atención. La recomendación de los profesionales de la salud impacta en la decisión de vacunarse. Se ha demostrado que el factor más importante asociado a la vacunación es la recomendación del equipo de salud independientemente de la percepción de la persona $^{(15)}$. Amaya y colaboradores realizaron una encuesta en el Centro Hospitalario Pereira Rossell, donde documentaron que solo $56 \%$ de los médicos entrevista- dos recomendaban la vacunación ${ }^{(16)}$. En este trabajo hubiese sido de interés conocer si los profesionales de salud del primer nivel de atención recomendaron la vacunación y si la hospitalización fue una oportunidad para recomendarla. Diferentes autores han documentado que la falta de consejería, promoción y tiempo en la consulta lleva a la pérdida de oportunidades de vacunación ${ }^{(15)}$. Actualmente la información sobre vacunas a través de medios de comunicación masivos y redes sociales son otros componentes importantes en la toma de decisiones de las personas. La comunicación sobre la vacuna contra el VPH ha generado rumores principalmente poniendo en duda su seguridad, influyendo en la negativa a vacunarse ${ }^{(14)}$. Debates actuales sobre la vacunación son cada vez más complejos, ya que más vacunas y combinaciones de vacunas están disponibles y los medios de comunicación global se han vuelto mucho más ubicuos, rápidos y no jerárquicos ${ }^{(17)}$.

Trabajos internacionales revelan que las familias con nivel socioeconómico y cultural más alto se mostraron más críticas frente a la información científica recibida a la hora de decidir entre vacunar o no a sus hijas ${ }^{(14)}$. Otros factores involucrados en la aceptabilidad de una vacuna referidos por la literatura, aunque no reportados en esta serie, son: la edad recomendada para la vacunación y las creencias religiosas. Los 12 años como edad recomendada para la vacunación gratuita en muchos países, incluido Uruguay, ha generado polémicas y barreras. Para muchas familias es difícil aceptar que las adolescentes comparten los mismos riesgos que mujeres que iniciaron relaciones sexuales, muchos padres consideran que aceptar la vacunación de sus hijas es legitimar conductas sexuales inapropiadas para la edad ${ }^{(14)}$. Sin embargo, se sabe que la vacunación contra el VPH produce una respuesta inmunitaria superior a la que produce la infección natural, existiendo una mayor producción de anticuerpos en menores de 15 años que en aquellas vacunadas después de esta edad ${ }^{(7)}$.

Los profesionales de la salud son responsables a su vez de asesorar sobre los beneficios de la vacunación y sus posibles efectos adversos. Deberían proporcionar información sobre educación sexual, métodos anticonceptivos y de barrera para la prevención de infecciones de trasmisión sexual y del embarazo no deseado, destacando la importancia de los controles en salud periódicos y la realización del tamizaje para el CCU, según las recomendaciones nacionales ${ }^{(18)}$.

En relación con la seguridad de la vacuna contra el VPH existen datos objetivos que permiten afirmar que se trata de una vacuna segura, que deberían ser transmitidos a la población general ${ }^{(19)}$. Los efectos adversos más frecuentes relacionados a esta vacuna son leves y autolimitados ${ }^{(9,19)}$. Los porcentajes de mujeres con 
eventos adversos graves, patologías clínicamente relevantes, nuevas enfermedades crónicas o nuevas enfermedades autoinmunes, fueron similares a la población no vacunada ${ }^{(20)}$. En esta serie no se reportaron efectos adversos graves.

Las hospitalizaciones son útiles para potenciar estrategias de educación y promoción en salud evitando oportunidades perdidas respecto a la actualización del carné de esquema de vacunación y promover inmunizaciones no sistemáticas. La disponibilidad, accesibilidad real, incluyendo la información sobre la vacunación contra el VPH, deben ser los pilares vectores de las estrategias orientadas a aumentar la cobertura vacunal.

\section{Conclusión}

La cobertura vacunal contra el VPH observada en esta serie fue similar a datos nacionales. La falta de información sobre la vacuna fue el motivo más frecuente de no vacunación reportado por las adolescentes. La hospitalización debe ser una oportunidad de prevención independientemente del motivo de ingreso.

\section{Agradecimientos}

A la Prof. Agda. Departamento de Farmacología y Terapéutica, Facultad de Medicina, Universidad de la República y asistente del Programa Nacional de Vacunaciones de Uruguay, Dra. Noelia Speranza, por sus aportes generosos para enriquecer el trabajo.

\section{Abstract}

Introduction: Uruguay offers a free HPV quadrivalent vaccine to adolescents 12 years of age or older. However, the vaccination rate was not as expected.

Objective: to describe the human papillomavirus (HPV) vaccination status, the reasons for non-vaccination and to report adverse effects in hospitalized adolescents in a reference center in Uruguay.

Materials and methods: descriptive study by means of an anonymous survey of adolescents of 12 years of age or older hospitalized in moderate care at Pereira Rossell Hospital Center during the second half of 2016. Adolescents who refused to participate were excluded from the study, as well as those with mental retardation and / or subject to palliative care. We analyzed: age, vaccination status against HPV, reasons for non-vaccination and vaccine hesitancy, and adverse effects. The research conducted was approved by the institution's ethics committee.

Results: the survey was answered by 112 adolescents who met the inclusion criteria. Mean age was 13.5 years old (12-14.6). 45/112 (40.1\%). Adolescents reported having received at least one dose of the HPV vaccine. The most frequent reason for non-vaccination was: lack of knowledge of the vaccine in 48 out of 67 $(71.6 \%)$, followed by rejection or rejection by the adolescent or responsible adult in 13 out of 67 (19.4\%). The main reason for refusal to receive the vaccine reported was: lack of information (7/13). No serious adverse events were reported.

Conclusions: vaccination coverage found was similar to national data. Knowledge and information of adolescents and their caregivers about this vaccine were the main reasons detected for non-vaccination.

\section{Resumo}

Introdução: no Uruguai una vacina tetravalente contra o Vírus do Papiloma Humano (VPH) é oferecida gratuitamente a todas as adolescentes com 12 ou mais anos. A cobertura obtida não foi a esperada.

Objetivo: descrever o estado vacinal contra o VPH, motivos de não vacinação e informação sobre efeitos adversos nas adolescentes hospitalizadas em um centro de referencia no Uruguai.

Materiais e métodos: estudo descritivo, realizado com questionário anônimo respondido por adolescentes com 12 ou mais anos, internadas em cuidados intermediários do Hospital Pediátrico do Centro Hospitalario Pereira Rossell, durante o segundo semestre de 2016. Foram excluídas as adolescentes que não quiseram participar, as portadoras de deficiência mental e/ou passiveis de cuidados paliativos. As seguintes variáveis foram analisadas: idade, estado vacinal contra VPH, motivos de não vacinação e de rejeição à vacina e efeitos adversos. O estudo foi aprovado pelo comité de ética da instituição.

Resultados: participaram 112 adolescentes com idade média 13,5 anos (12-14,6). 45 informaram ter recebido pelo menos uma dose da vacina contra VPH (45/112 - 40,1\%). O motivo de não vacinação mais frequente foi o desconhecimento da existência da vacina 48/67 (71,6\%), seguido de rejeição ou negativa da adolescente ou do adulto responsável 13/67 (19,4\%). O motivo principal de rejeição a ser vacinada foi a falta de informação 7/13. Não foram registrados efeitos adversos graves.

Conclusões: a cobertura vacinal registrada foi similar aos dados nacionais. O conhecimento e a informação das adolescentes e de seus cuidadores sobre esta vacina foram os principais motivos de não vacinação detectados.

\section{Bibliografía}

1. Comisión Honoraria de lucha contra el cáncer. Registro Nacional de Cáncer. Cáncer de cuello uterino en Uruguay. Montevideo: CHLCC, 2016. Disponible en: http://www.co- 
misioncancer.org.uy/categoria_53_1.html. [Consulta: 13 enero 2018].

2. Berois N, De Cremoux P, Mazal D, Sica A, Cedeira M, Caserta B, et al. Prevalence and Distribution of High-Risk Human Papillomavirus Genotypes in Invasive Carcinoma of the Uterine Cérvix in Uruguay. Int J Gynecol Cancer 2013; 23(3):527-32.

3. Berois N, Heard I, Fort Z, Alonso R, Sica A, Moerzinger $\mathbf{P}$, et al. Prevalence of Type-Specific HPV Infection in Uruguay. J Med Virol 2014; 86(4):647-52.

4. Sam S, Ortiz de la Peña y Carranza A, Lira J. Virus del papiloma humano y adolescencia. Ginecol Obstet Mex 2011; 79(4):214-24. Disponible en: www.medigraphic.com/pdfs/ginobsmex/gom-2011/gom114g.pdf. [Consulta: 13 enero 2018].

5. Pizzocaro G, Algaba F, Solsona E, Tana S, Van der Poel H, Watkin N, et al. Guía clínica sobre el cáncer de pene. Arnhem: European Association of Urology, 2010. Disponible en: http://www.aeu.es/UserFiles/05-GUIA_CLINICA_ CANCER_PENE.pdf. [Consulta: 12 enero 2018].

6. García A. Infecciones de la vulva con virus del papiloma humano. Arch Med Actual Trac Gen Inf 2011; 3(5):30-1. Disponible en: http://www.medigraphic.com/pdfs/archivostgi/tgi-2011/tgi115f.pdf. [Consulta: 12 enero 2018].

7. Ochoa F, Guarneros de Regil D, Velasco M. Infección por virus del papiloma humano en mujeres y su prevención. GAMO 2015; 14(3):157-63. Disponible en: https://www. sciencedirect.com/science/article/pii/S1665920115000607. [Consulta: 10 enero 2018].

8. Asociación Española de Patología Cervical y Colposcopía. Guías de prevención de cáncer de cuello de útero 2014. Madrid: AEPCC, 2015. Disponible en: http://www.aepcc.org/wp-con-

tent/uploads/2016/01/AEPCC_revista02.pdf. [Consulta: 12 enero 2018].

9. Pérez G, Frantchez V, Medina J. La vacuna contra el virus del papiloma humano es segura y eficaz. Montevideo: Universidad de la República. Facultad de Medicina. Cátedra de Enfermedades Infecciosas, 2016. Disponible en: http://www.infectologia.edu.uy/divulgacion-medica/novedades-y-avances/la-vacuna-contra-el-virus-del-papiloma-humano-es-segura-y-eficaz. [Consulta: 10 enero 2018].

10. Ministerio de Salud Pública. Guía de práctica clínica de tamizaje de cáncer de cuello uterino. Montevideo: MSP, 2014. Disponible en: http://www.msp.gub.uy/sites/default/files/archivos_adjuntos/Gu\%C3\%ADa\%20cuello\%20uterino\%20MSP\%202014_1.pdf. [Consulta: 11 enero 2018].

11. Pinto B, Gulfo R, Sanabria A, Sánchez S, Mojica MC, Endo J. Vacunación obligatoria y movimientos anti-vacuna: algunas propuestas desde la bioética. Ea Journal 2012; 4(2):1-29. Disponible en: http://www.ea-journal.com/ima-
ges/stories/Art04.02/Pinto-et-al-Vacunacion-obligatoria-y-movimiento-anti-vacuna.pdf. [Consulta: 10 enero 2018].

12. Seman C. Análisis de las políticas públicas de prevención del cáncer de cuello uterino vinculadas al control del virus del papiloma humano (VPH) en Argentina. Buenos Aires: Programa de Salud Pública en Ambientes Urbanos, 2016. Disponible en: http://digitalcollections.sit.edu/cgi/viewcontent.cgi?article $=3443 \&$ context $=$ isp_collection. [Consulta: 10 enero 2018]

13. Ng J, Ye F, Roth L, Sobel K, Byron S, Barton M, et al. Human papillomavirus vaccination coverage among female adolescents in managed care plans: United States, 2013. Morb Mortal Wkly Rep 2015; 64(42):1185-9. Disponible en: https://www.cdc.gov/mmwr/pdf/wk/mm6442.pdf. [Consulta: 12 enero 2018].

14. Wiesner C, Piñeros M, Trujillo L, Cortés C, Ardilla J. Aceptabilidad de la vacuna contra el Virus Papiloma Humano en padres de adolescentes, en Colombia. Rev. salud pública 2010; (12)6:961-73. Disponible en: http://www.scielo.org.co/pdf/rsap/v12n6/v12n6a08.pdf. [Consulta: 4 enero 2018].

15. Pérez G, Brasó P, Medina J. Conocimiento de los médicos especialistas o en formación acerca de vacunas no sistemáticas en Uruguay. Rev Méd Urug 2017; 33(1):34-46. Disponible en: http://www.rmu.org.uy/revista/2017v1/art5.pdf. [Consulta: 13 enero 2018].

16. Amaya G, Romero C, Veirano C, Barreto A, Pírez MC. ¿Cuánto saben los médicos sobre vacunas no sistemáticas? IV Congreso Internacional de Infectología Pediátrica y Vacunas. Buenos Aires: SADIP, 2014.

17. Larson HJ, Jarrett C, Eckersberger E, Smith DM, Paterson $P$. Understanding vaccine hesitancy around vaccines and vaccination from a global perspective: a systematic review of published literature, 2007-2012. Vaccine 2014; 32(6):2150-9.

18. Pedraza A. Aspectos conjuntos profesionales pediatría/enfermería. El equipo pediátrico de atención primaria: aspectos formativos y asistenciales. Rev Pediatr Aten Primaria Supl 2011; 13(20):71-80. Disponible en: http://scielo.isciii.es/pdf/pap/v13s20/mesa06.pdf. [Consulta: 10 enero 2018].

19. Viroga S, Speranza N. Seguridad de la vacuna VPH: ¿qué sabemos y qué hay de nuevo? Bol Farmacol 2016; 7(1):1-6. Disponible en: http://www.boletinfarmacologia.hc.edu.uy/ images/stories/boletin/seguridad_de_la_vacuna_vph.pdf. [Consulta: 7 enero 2018].

20. Bruni L, Serrano B, Bosch X, Castellsagué X. Vacuna frente al virus del papiloma humano: eficacia y seguridad. Enferm Infecc Microbiol Clin 2015; 33(5):342-54. 